\title{
Selective Functionalization of Microstructured Surfaces by Laser-Assisted Particle Transfer
}

Clemens von Bojnicic-Kninski, Valentina Bykovskaya, Frieder Maerkle, Roman Popov, Andrea Palermo, Daniela S. Mattes, Laura K. Weber, Barbara Ridder, Tobias C. Foertsch, Alexander Welle, Felix F. Loeffler, Frank Breitling, and Alexander Nesterov-Mueller*

C. von Bojnicic-Kninski, V. Bykovskaya, R. Popov, A. Palermo, D. S. Mattes, L. K. Weber, B. Ridder, T. C. Foertsch, Dr. F. F. Loeffler, Prof. Dr. F. Breitling

Karlsruhe Institute of Technology

Institute of Microstructure Technology

Hermann-von-Helmholtz-Platz 1, 76344

Eggenstein-Leopoldshafen, Germany

Dr. A. Welle

Karlsruhe Institute of Technology

Institute of Functional Interfaces

Hermann-von-Helmholtz-Platz 1, 76344

Eggenstein-Leopoldshafen, Germany

Dr. F. Maerkle

Badener Straße 7, 76337

Waldbronn, Germany

[*] Prof. Dr. Alexander Nesterov-Mueller

Karlsruhe Institute of Technology

Institute of Microstructure Technology

Hermann-von-Helmholtz-Platz 1, 76344

Eggenstein-Leopoldshafen, Germany

E-mail: alexander.nesterov-mueller@kit.edu

Keywords: laser, microstructures, functional microparticles, functionalization, solid phase synthesis

Microcavity arrays represent millions of different reaction compartments to screen for e.g. molecular interactions, exogenous factors for cells or enzymatic activity. We present a novel method to selectively synthesize different compounds in arrays of microcavities with up to $1,000,000$ cavities per $\mathrm{cm}^{2}$. In our approach, polymer microparticles with embedded preactivated monomers are selectively transferred into microcavities with laser radiation. After particle patterning, heating of the particle matrix simultaneously leads to diffusion and coupling of the monomers inside each microcavity separately. This method exhibits flexibility, 
not only in the choice of compounds, but also in the choice of particle matrix material, which determines the chemical reaction environment. The laser-assisted selective functionalization of microcavities can be easily combined with the intensively growing number of laser applications for patterning of molecules and cells, which is useful for the development of novel biological assays.

\section{Introduction}

Microcavity-structured surfaces offer a range of different applications, especially in highthroughput screenings of millions of molecular interactions, such as oligonucleotides. ${ }^{[1]}$ Recently, microstructures were also used to analyze single cells ${ }^{[2]}$ or to screen the functional heterogeneity of enzyme activity on single molecule level, ${ }^{[3]}$ as well as studying the impact of geometrical constraints and ligand specificity on stem cells. ${ }^{[4]}$

Although the technology for combinatorial chemistry, based on the solid phase synthesis principle of Merrifield, ${ }^{[5]}$ is well developed, especially for peptide arrays, ${ }^{[6]}$ the functionalization of microcavities in the ultra-high density array format remains challenging. Spotting of solubilized reagents either leads to spreading of the solvents on the surface or the minute amounts of solvent rapidly evaporate. Also lithographic synthesis methods, ${ }^{[7]}$ based on the light-directed cleavage of photolabile protecting groups, exhibit principle drawbacks: First, a complex microstructured and microfluidic substrate is required, to prevent the diffusion of light-generated deprotecting solution to adjacent cavities. Secondly, each monomer requires an individual coupling step so that repetitive coupling is necessary, making the process very time-consuming. ${ }^{[8]}$ Particle-based methods for the transport of monomers, such as xerographic approaches $^{[9]}$ or selective laser melting, ${ }^{[10]}$ overcome these drawbacks, but they are not applicable for the functionalization of microcavities in the ultra-high density array format. 
These particle deposition techniques are limited to 800 spots per $\mathrm{cm}^{2}$ for xerographic approaches $^{[11]}$ and 40,000 spots per $\mathrm{cm}^{2}$ for combinatorial laser fusing. ${ }^{[10]}$

Here, we present a laser-assisted particle transfer method for the selective functionalization of up to 1 million microcavities per $\mathrm{cm}^{2}$. In our approach, we use styrene acrylic copolymer microparticles with embedded pre-activated monomers, which are selectively transferred into microcavities of a microstructured glass surface. After particle patterning, heating of the particle matrix leads to diffusion of the pre-activated monomers inside the polymer matrix and coupling to a functionalized surface. We were able to significantly increase the density of patterns by transferring particles from microstructured donor surfaces to a microstructured acceptor surface, which restricts the particle transfer to a defined volume, prohibiting unwanted particle spreading.

\section{Selective functionalization of microcavities}

\subsection{Principle}

To create a selective microparticle pattern, we use microstructured glass slides. The cylindrical microcavities were manufactured in glass via dry-etching (Fused Silica, Siegert Wafer GmbH) (Figure $1 \mathrm{a}+\mathrm{b})$. A donor and an acceptor slide are positioned on top of each other, so that the microstructures are facing each other and the centers of the opposing cavities coincide. ${ }^{[12]}$ The donor slide is filled with microparticles, which contain activated monomers. The microcavities of the donor slide allow for an easy, precise, and uniform distribution of the particles across the surface. Additionally, the cavities serve as a gun barrel to guide the particles from the donor cavities into the underlying cavities of the acceptor (synthesis) slide, while being transferred by laser radiation (Figure 1 c1).

To transfer the particles, a focused laser pulse is used to propel the particles out of a donor slide cavity into a microcavity of the synthesis slide. Each of these cavities acts as a tiny 
synthesis reactor, prohibiting the diffusion of monomers to adjacent cavities. By replacing the donor slide with another donor, containing a different type of monomer particles, and repeating the transfer process, a combinatorial pattern of particles can be generated in the synthesis slide (Figure 1 c2).

\subsection{Slide layouts}

We present two methods for the particle transfer, resulting in different spot densities ranging from $111,111 \mathrm{~cm}^{-2}$ up to $1,000,000 \mathrm{~cm}^{-2}$. Transfer method \#1 uses orthogonally arranged donor and synthesis cavities with the same pitch, but different diameters (Figure $1 \mathrm{~d}$ ). This structure combination demands for a precise alignment of the donor slide on top of the synthesis slide in translation as well as in angle to achieve coinciding cavity centers. To increase the tolerances during this alignment, the donor cavities have a smaller diameter $\left(d_{\text {Donor }}=0.7 \cdot d_{\text {Synthesis }}\right)$ than the synthesis cavities, whereby a small offset and tilt of the donor slide in respect to the synthesis slide can be compensated. Another effect of the smaller donor cavities is the reduced scattering during the transfer, since scattered particles are collected by the bigger synthesis cavity acting like a funnel. With this arrangement we achieved spot densities of 444,444 $\mathrm{cm}^{-2}$ and 1,000,000 $\mathrm{cm}^{-2}$. Transfer method \#2 does not require the alignment of donor and synthesis cavities. By arranging the donor cavities in a hexagonal manner and making them much smaller than the synthesis cavities $\left(d_{\text {Donor }}=0.3 \cdot d_{\text {Synthesis }}\right)$, multiple small donor cavities are located on top of one big synthesis cavity (Figure 1 e). However, this arrangement only allows for a lower spot density, defined by the minimum diameter of the donor cavity of $5 \mu$ m (in order to apply transfer method \#2 to synthesis cavities with a diameter of $7 \mu \mathrm{m}$, the donor cavities would need a diameter of $2.1 \mu \mathrm{m}$, which could carry only a single particle) and the fact that at least three donor cavities should be 
completely above one synthesis cavity to ensure a sufficient particle transfer. Using this arrangement, we achieved a spot density of $111,111 \mathrm{~cm}^{-2}$.

\subsection{Donor slide}

To achieve a reproducible transfer result, the donor slide has to be coated with a light absorbing layer prior to the deposition of the particles. By absorbing the laser radiation within this additional layer (in literature often referred to as the dynamic release layer), the required laser intensity to perform the transfer is reduced. Furthermore, it protects the functional particles from being damaged by the laser radiation. ${ }^{[13]}$

With a thickness of $15 \mathrm{~nm}$ the applied gold layer is thicker than the penetration depth of the laser of $532 \mathrm{~nm}$ wavelength, which leads to the complete absorption of the laser radiation. ${ }^{[14]}$ The absorbed energy causes the instantaneous evaporation of the gold layer, and the vapor pressure propels the particles towards the synthesis slide. ${ }^{[15]}$

\section{Materials and methods}

\subsection{Optical setup for particle transfer between two microstructured slides}

We use a frequency doubled diode-pumped Nd:YAG laser with a wavelength of $532 \mathrm{~nm}$ and a maximum pulse energy of $350 \mu \mathrm{J}$ (MATRIX 532-7-30, Coherent, Inc.) to perform the particle transfer. The laser is guided into an inverse microscope (Axiovert 35, VEB Carl Zeiss Jena) and focused to a minimum focal spot diameter of $5.1 \mu \mathrm{m}$ at $1 / \mathrm{e}^{2}$ in intensity on the probes using a 20x objective (LMH-20X-532, Thorlabs, Inc.) (Figure $2 \mathrm{a}+\mathrm{c}$ ). To exactly position the donor cavities on top of the synthesis cavities with coinciding centers, a mechanical slide positioning system was designed, allowing the donor slide to be positioned laterally and rotated with respect to the synthesis slide (Figure $2 \mathrm{~b}+\mathrm{e}$ ). To minimize the distance between 
their surfaces to $7 \pm 3 \mu \mathrm{m}$, the two slides are pressed together by springs. The slide positioning system is mounted onto a motorized XY-table (SCAN-IM 130x100, Märzhäuser Wetzlar GmbH \& Co. KG), so that the laser transfer can be carried out automatically by a computer, moving the samples and triggering the laser pulses. Due to the springs pressing the two slides together, the slides are not orthogonal to the incident laser beam. Therefore, to adjust the precise vertical position of the laser focus in respect to the samples, we have mounted a moveable lens (LA1608, Thorlabs Inc.) before the focusing objective (Figure $2 \mathrm{~d}$ ). Measurements show that a maximum movement of the focal plane of $140 \mu \mathrm{m}$ is needed to compensate for the tilt induced by the springs of the slide positioning system (Supporting Information: Tilt of focal plane). This movement is controlled by moving the lens along the laser beam path with an actuator. By moving the lens for $1 \mathrm{~mm}$, the position of the focal plane changes by $13.9 \mu \mathrm{m}$. To enable this refocusing, the inclination of the focal plane is measured in advance. The resulting plane equation is solved for every spot position in order to calculate the shift in intensity. If this shift is larger than a set threshold (10\%), the laser is refocused by moving the lens.

\subsection{Transfer process}

The light absorbing layer on the donor slide consists of a $15 \mathrm{~nm}$ thick gold layer, sputtered (MED 010, Balzers Union) onto the complete surface of the slide. Since this layer is only needed within the cavities, the upper surface of the donor slide is polished using a lint-free tissue such that the gold layer only remains inside the cavities (Figure $3 \mathrm{a} 2+\mathrm{b}$ ). To fill the microparticles into the microcavities, we place a small amount of particles as a powder on the substrate using a spatula (15 - $20 \mathrm{mg})$ and gently wipe them in with a lint-free tissue. Thereby, particles are pushed into the cavities, but at the same time are removed from the top surface (Figure $3 \mathrm{a} 3+\mathrm{c}$ ). 
The microparticles are composed of $88 \%(\mathrm{w} / \mathrm{w})$ polymer matrix (SLEC PLT-7552, Sekisui Chemical GmbH), $10 \%$ (w/w) activated monomers and $2 \%(\mathrm{w} / \mathrm{w})$ graphite nanoparticles. We used two kinds of monomers, Fmoc (9-fluorenylmethoxycarbonyl)-protected glycine monomer pre-activated as OPfp (pentafluorophenyl) ester and biotin-OPfp. The graphite nanoparticles increase the laser absorption of the particles, which shield the sensitive synthesis slide surface from the laser radiation. Produced in a spray drying process (B 290, BÜCHI Labortechnik GmbH), the particles have a spherical shape with a median diameter of $3.3-3.5 \mu \mathrm{m}$ (Figure $3 \mathrm{f}+\mathrm{g}){ }^{[16]}$

During the transfer process, these particles overcome the distance of $7 \pm 3 \mu \mathrm{m}$ between the donor and the synthesis slide according to their stopping distance (Supporting Information: Stopping distance). However, they lead to a wide spreading and therefore only allow for a reduced spot density. To increase the lateral resolution of transferred particles, the donor slide is heated for $15 \mathrm{~min}$ at $90^{\circ} \mathrm{C}$ under an inert argon atmosphere to sinter the deposited particles, so that in each cavity the particles agglomerate (Figure $3 \mathrm{a} 4+\mathrm{d}$ ). The inert atmosphere is needed to prevent the activated monomers to react with oxygen of the surrounding atmosphere. By sintering the particles to agglomerates, we are able to reach spot densities of up to $1,000,000 \mathrm{~cm}^{-2}$. Furthermore, bigger particles have a larger stopping distance, which is beneficial for the transfer. The sintered donor slides are used for the transfer (Figure 3 a5). Preparation of the donor slides requires $45 \mathrm{~min}$, whereas multiple donor slides can be processed in parallel. The alignment of the donor slide on top of the synthesis slide can be performed within 10 min using the slide positioning system.

\subsection{Synthesis slide}

Onto the surface of the synthesis slide an approx. $25 \mathrm{~nm}$ thick layer of copolymer, containing $10 \%$ (n/n) poly(ethylene glycol) methacrylate (PEGMA) and $90 \%$ (n/n) methyl methacrylate 
(MMA) was grafted by surface-initiated Atom Transfer Radical Polymerization. ${ }^{[17]}$ This polymer coating provides for the functional hydroxyl groups, which are subsequently modified with three $\beta$-alanine residues to render the amino groups required for the coupling of monomers, brought into the synthesis cavities by the particles. ${ }^{[18]}$

\subsection{Synthesis}

After generation of the microparticle pattern in the cavities, the synthesis slide is heated in an oven to $90{ }^{\circ} \mathrm{C}$ for 60 min under argon atmosphere, initiating the coupling reaction: the particle matrix material softens, the embedded monomers diffuse to the surface and couple to the surface-bound amino groups (Figure 4 b). ${ }^{[19]}$ Once the slide has cooled down to room temperature, it is washed in acetone (95 \% v/v) and methoxyethylamine ( $5 \% \mathrm{v} / \mathrm{v})$ to remove the particle matrix material and uncoupled monomers. Remaining free amino groups of the copolymer layer are capped with a solution of acetic anhydride $(10 \% \mathrm{v} / \mathrm{v})$, diisopropylethylamine (20\% v/v), and dimethylformamide (70 \% v/v). This results in inactivation (acetylation) of unreacted surface-bound amino groups, prohibiting unwanted reactions in the next steps.

To stain the coupled monomers with a fluorescent dye, we first remove the Fmoc protecting groups of glycine with a solution of piperidine $(20 \% \mathrm{v} / \mathrm{v})$ in dimethylformamide $(80 \% \mathrm{v} / \mathrm{v})$

(Figure 4 c). Thereafter, the synthesis slide is incubated with DyLight $650 \mathrm{~N}$ Hydroxysuccinimid ester (NHS ester) in Phosphate Buffer Saline - Tween 20 (PBS-T buffer) (9.4 $\mu \mathrm{mol} \mathrm{L}{ }^{-1}$ ) to couple DyLight 650 to the free amino groups of the surface-bound glycine. Subsequently, the surface is incubated with Streptavidin DyLight 550 in PBS-T buffer and Rockland Blocking Buffer (1.7 nmol L $\left.{ }^{-1}\right)$, to label the biotin spots (Figure $4 \mathrm{~d}$ ). For fluorescent imaging of the microstructured slides, we used a confocal microscope (TCS SP-5, Leica Microsystems). 


\section{Results}

We have studied three different combinations of donor and synthesis cavity sizes (Supporting information: Structure combinations). For the synthesis slides with cavity diameters of $7 \mu \mathrm{m}$ and $11 \mu \mathrm{m}$ (spot densities of 1,000,000 $\mathrm{cm}^{-2}$ and 444,444 $\mathrm{cm}^{-2}$ ), the donor cavities had the same pitch as the donor cavities, but a smaller diameter of $5 \mu \mathrm{m}$ and $8 \mu \mathrm{m}$ (transfer method \#1). In case of synthesis cavities with a diameter of $25 \mu \mathrm{m}\left(111,111 \mathrm{~cm}^{-2}\right)$, we used a donor slide with a very dense hexagonal arrangement of small cavities with a diameter of $7 \mu \mathrm{m}$ according to transfer method \#2, which requires no alignment of the donor to the synthesis slide. Instead, a defocused laser pulse depletes multiple donor cavities at once and transfers the particles into the respectively much bigger synthesis cavity below. Here, only the position of the laser focus relative to the synthesis slide has to be controlled.

Previous experiments showed that the particles tend to stick to the cavity walls during the coupling step in the oven, if an aspect-ratio $>1$ (ratio of depth to cavity diameter) was used. To assure that the particles stick to the bottom of the cavities and not to the cylinder surface, we have chosen a cavity aspect ratio of $<1$ for the synthesis slide (Supporting Information: Aspect ratio).

During irradiation with the laser, the donor cavities are emptied completely (Figure 5 a). We also observe that the sputtered gold layer is completely removed, which supports the assumption, that the gold is vaporized. In addition, we could not detect gold fragments on the synthesis slide after the particle transfer using a scanning electron microscope (SEM) (Figure 5 b). Yet, the gold vapor seems to condensate on the surface of the synthesis cavities, which was detected via secondary ion mass spectrometry (SIMS) (Figure 5 c). To obtain the ToFSIMS measurement, an area of 13 by 13 cavities was filled using the transfer method \#2. 
Even though high temperatures are reached within the laser focal point, the particles as well as the embedded monomers are not damaged and are still active. By SEM analysis of the transferred particles, we could verify that the particles maintained their spherical shape, while no pieces of destroyed particles could be found. Furthermore, the functionality of the monomers is still given, which is shown in the fluorescent scans of the coupled and stained monomers (Figure 6).

For the described experiments, we have qualitatively characterized the reproducibility of the particle transfer $R_{\text {trans }}$ and the scattering $S_{\text {trans. }}$ The reproducibility $R_{\text {trans }}$ was derived from observing light microscopic images of the transferred particles (Figure $7 \mathrm{a}-\mathrm{d}$ ). By comparing the ideal pattern with the actually transferred pattern, the reproducibility was defined as the ratio of actually filled synthesis cavities to theoretically filled synthesis cavities. We could achieve a reproducibility of $99.9 \%$ for the spot densities of $111,111 \mathrm{~cm}^{-2}$ and $444,444 \mathrm{~cm}^{-2}$ and a reproducibility of $99.5 \%$ for the ultra-high spot density of 1,000,000 $\mathrm{cm}^{-2}$ (Supporting Information: Table S6). The lower reproducibility for a pitch size of $10 \mu \mathrm{m}$ can be explained with the tolerance limits of our laser system. The position of the laser focus on the slide has an accuracy of $\pm 1 \mu \mathrm{m}$, as well as the motorized microscope stage. Together, this leads to a maximum error of $2 \mu \mathrm{m}$. Thus, the laser focus is occasionally not positioned correctly in the middle of a cavity, leading to fluctuations of the absorbed laser energy. If the energy is too low, the laser does not ablate the gold within the donor cavities and no transfer occurs.

The scattering $S_{\text {trans }}$ was defined as the volume of scattered particles divided by the volume of the total transferred particles (Figure $7 \mathrm{e}-\mathrm{h}$ ). For all three examined spot densities, the scattering $\mathrm{S}_{\text {trans }}$ was below $4 \%$ (Supporting Information: Table S7). The scattering did not lead to contaminations in neighboring structures, but only on the top of the bridge parts of the microstructures. 
According to the synthesis protocol, we conducted the coupling reaction of the glycine and biotin monomers with subsequent fluorescent staining after the particle patterning. In the fluorescent images, biotin spots appear inhomogeneously stained, whereas glycine spots appear homogeneously stained (Figure 6). This originates from different dissolving behaviors of the two monomers in the polymer matrix of the microparticles. Whereas glycine dissolves in the polymer matrix at a concentration of $10 \%(\mathrm{w} / \mathrm{w})$, biotin forms agglomerates within the particles at a similar concentration. This fact is reflected in the smaller fluorescent signal variation $v$ for glycine spots of $<7 \%$ in comparison to $v<19 \%$ for biotin spots (Figure $6 \mathrm{~d}$ ). The histogram of all spots of the two channels illustrates this variation by two very different standard deviations (Figure 6 e). Furthermore, we calculated the signal to noise ratio (SNR), defined as the ratio between the average value of fluorescent spots of glycine and biotin and the standard deviation of empty spots, for all three spot densities (Figure $6 \mathrm{f}$ ). In all three geometry types, the SNR exhibits relatively large values of $>15$. The statistical analyses were conducted with the average and median values of spot intensities assigned to each spot (Supporting Information: Statistical analysis).

\section{Conclusion}

To our knowledge, we have demonstrated the selective functionalization of the smallest microcavities so far. We have generated particle patterns with an amino acid and biotin. The presented technological platform exhibits flexibility in the spot density, ranging from $111,111 \mathrm{~cm}^{-2}$ up to $1,000,000 \mathrm{~cm}^{-2}$. According to these spot densities, the system used to selectively functionalize microcavities can process cavity diameters ranging from $25 \mu \mathrm{m}$ down to $7 \mu \mathrm{m}$. For larger cavities, spotting of the monomers from solution may be preferable, as the spreading of the spotting solution in this scale will not contaminate adjacent cavities. 
We proposed two similar transfer methods, which allow for either ultra-high spot densities or simplicity during the alignment process. The physical transfer as well as the chemical coupling and staining proved the applicability of the selective functionalization. Smaller cavity diameter of less than $7 \mu \mathrm{m}$ turned out to be not applicable for the described methods for three reasons: First, the alignment of such small structures demands for very tight tolerances, second the laser focus diameter needs to fit the smaller cavities and third, the scattering of particles becomes problematic, since they also scatter into adjacent cavities and induce contaminations.

Making use of the thresholding of the laser, which refocuses the laser according to the identified geometrical orientation of the focal plane, the necessary time to lase one spot plus moving the XY-table to the next spot currently takes $250 \mathrm{~ms}$. To selectively functionalize 10,000 cavities with 5 different monomers it takes $8 \mathrm{~h}$ including the preparation of donor slides (3.5 h) and chemistry (3 h excluding the staining of coupled monomers). The transfer itself lasts $40 \mathrm{~min}$. To accelerate the transfer process, instead of positioning the XY-table, a galvanic mirror scan head could be used, which would significantly decrease the processing time per cavity to $<20 \mathrm{~ms}$, which reduces the time for the transfer down to $3 \mathrm{~min}$.

The major advantage of our densely arranged microcavities is the ultra-high spot density, which allows on the one hand screening of millions of interactions in one experiment and on the other hand to allow for the unprecedentedly low consumption of analyte required to screen the synthesized compounds. This can be very important for high-throughput analysis of precious samples in biomedical research. 


\section{Supporting Information}

Supporting Information is available from the Wiley Online Library or from the author.

\section{Acknowledgements}

We thank Dr. Bastian Münster and Dr. Jakob Striffler for their help with the particle and synthesis slide preparation as well as Alexandra Moritz for the technical assistance. The ToFSIMS measurements were performed within the KNMF Laboratory for Spectroscopy and Microscopy, Karlsruhe Institute of Technology. This work was supported by funds from the ERC (St Grant no. 277863), the HRJRG (grant no. 316) and the BMBF (grant no. 031A170A, 03EK3030A, 031A095C).

\section{References}

[1] K. L. Gunderson, Genome Research 2004, 14, 870.

[2] M. Hosokawa, A. Arakaki, M. Takahashi, T. Mori, H. Takeyama, T. Matsunaga, Anal. Chem. 2009, 81, 5308.

[3] R. B. Liebherr, M. Renner, H. H. Gorris, J. Am. Chem. Soc. 2014, 136, 5949.

[4] I. Kurth, K. Franke, T. Pompe, M. Bornhäuser, C. Werner, Macromol. Biosci. 2011, 11, 739.

[5] R. B. Merrifield, J. Am. Chem. Soc. 1963.

[6] C. Katz, L. Levy-Beladev, S. Rotem-Bamberger, T. Rito, S. G. D. Rüdiger, A. Friedler, Chem. Soc. Rev. 2011, 40, 2131.

[7] S. Fodor, J. Read, M. Pirrung, L. Stryer, A. Lu, D. Solas, Science 1991, 251, 767.

[8] F. F. Loeffler, Y.-C. Cheng, B. Muenster, J. Striffler, F. C. Liu, F. Ralf Bischoff, E. Doersam, F. Breitling, A. Nesterov-Mueller, in Fundamentals and Application of New Bioproduction Systems, Vol. 137 (Ed.: A.-P. Zeng), Springer Berlin Heidelberg. Berlin, Heidelberg 2013, p. 1. 
[9] M. Beyer, A. Nesterov, I. Block, K. Konig, T. Felgenhauer, S. Fernandez, K. Leibe, G. Torralba, M. Hausmann, U. Trunk, V. Lindenstruth, F. R. Bischoff, V. Stadler, F. Breitling, Science 2007, 318, 1888.

[10] F. Maerkle, F. F. Loeffler, S. Schillo, T. Foertsch, B. Muenster, J. Striffler, C. Schirwitz, F. R. Bischoff, F. Breitling, A. Nesterov-Mueller, Adv. Mater. 2014, 26, 3730.

[11] V. Stadler, T. Felgenhauer, M. Beyer, S. Fernandez, K. Leibe, S. Güttler, M. Gröning, K. König, G. Torralba, M. Hausmann, V. Lindenstruth, A. Nesterov, I. Block, R. Pipkorn, A. Poustka, F. R. Bischoff, F. Breitling, Angew. Chem. Int. Ed. 2008, 47, 7132.

[12] S. Eun Chung, J. Kim, D. Yoon Oh, Y. Song, S. Hoon Lee, S. Min, S. Kwon, Nature communications 2014, 5, 3468.

[13]N. T. Kattamis, P. E. Purnick, R. Weiss, C. B. Arnold, Appl. Phys. Lett. 2007, 91, 171120.

[14] K. Sugioka, M. Meunier, A. Piqué, Laser precision microfabrication, Springer-Verlag, Heidelberg, New York 2010.

[15] L. Rapp, C. Cibert, S. Nénon, A. P. Alloncle, M. Nagel, T. Lippert, C. VidelotAckermann, F. Fages, P. Delaporte, Applied Surface Science 2011, 257, 5245.

[16] B. Muenster, A. Welle, B. Ridder, D. Althuon, J. Striffler, T. C. Foertsch, L. Hahn, R. Thelen, V. Stadler, A. Nesterov-Mueller, F. Breitling, F. F. Loeffler, Applied Surface Science 2016, 360, 306.

[17] V. Stadler, R. Kirmse, M. Beyer, F. Breitling, T. Ludwig, F. R. Bischoff, Langmuir 2008, 24, 8151.

[18] A. Nesterov, E. Dörsam, Y.-C. Cheng, C. Schirwitz, F. Märkle, F. Löffler, K. König, V. Stadler, R. Bischoff, F. Breitling, Small molecule microarrays: methods and protocols 2010.

[19] F. Breitling, T. Felgenhauer, A. Nesterov, V. Lindenstruth, V. Stadler, F. R. Bischoff, ChemBioChem 2009, 10, 803. 


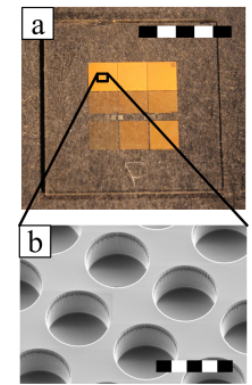

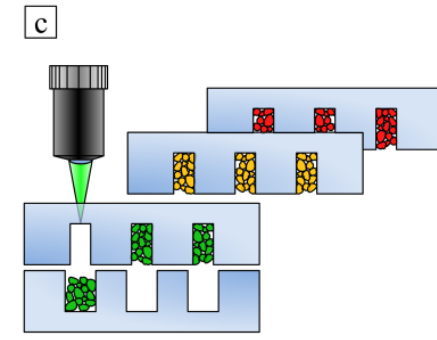

(1)
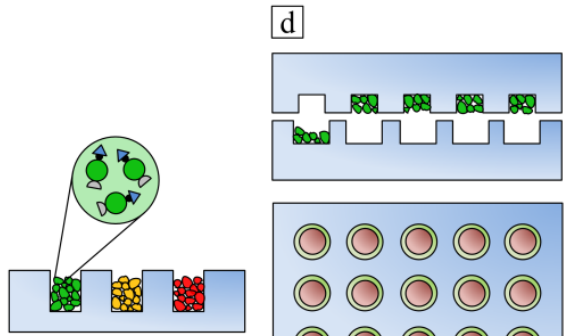

(2)

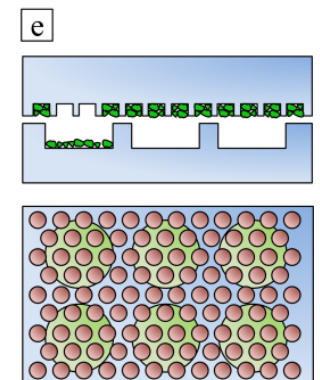

Figure 1. Laser-assisted particle transfer. (a) Microstructured glass slide (scale bar $10 \mathrm{~mm}$ ) and (b) Scanning electron microscope image of cavities with a diameter of $15 \mu \mathrm{m}$, a pitch of $20 \mu \mathrm{m}$ and a depth of $10 \mu \mathrm{m}$ (scale bar $20 \mu \mathrm{m}$ ) (c) Schematic physical deposition process: Two microstructured glass slides are positioned on top of each other. A focused laser beam transfers microparticles with embedded monomers from the top slide (donor slide) to the bottom slide (synthesis slide) (c1). This patterning process is repeated with different donor slides to achieve a combinatorial pattern in the synthesis slide (c2). The embedded monomers are common derivatives for solid phase chemistry as illustrated in Figure 4. (d) Transfer method \#1 - the donor and synthesis cavities share the same pitch, while the donor cavities are smaller than the synthesis cavities. One donor cavity is located above one synthesis cavity. (e) Transfer method \#2 - the donor cavities are arranged in a hexagonal manner and are much smaller than the synthesis cavities. Using this structure combination, no alignment of the donor cavities on top of the synthesis cavities is necessary. 

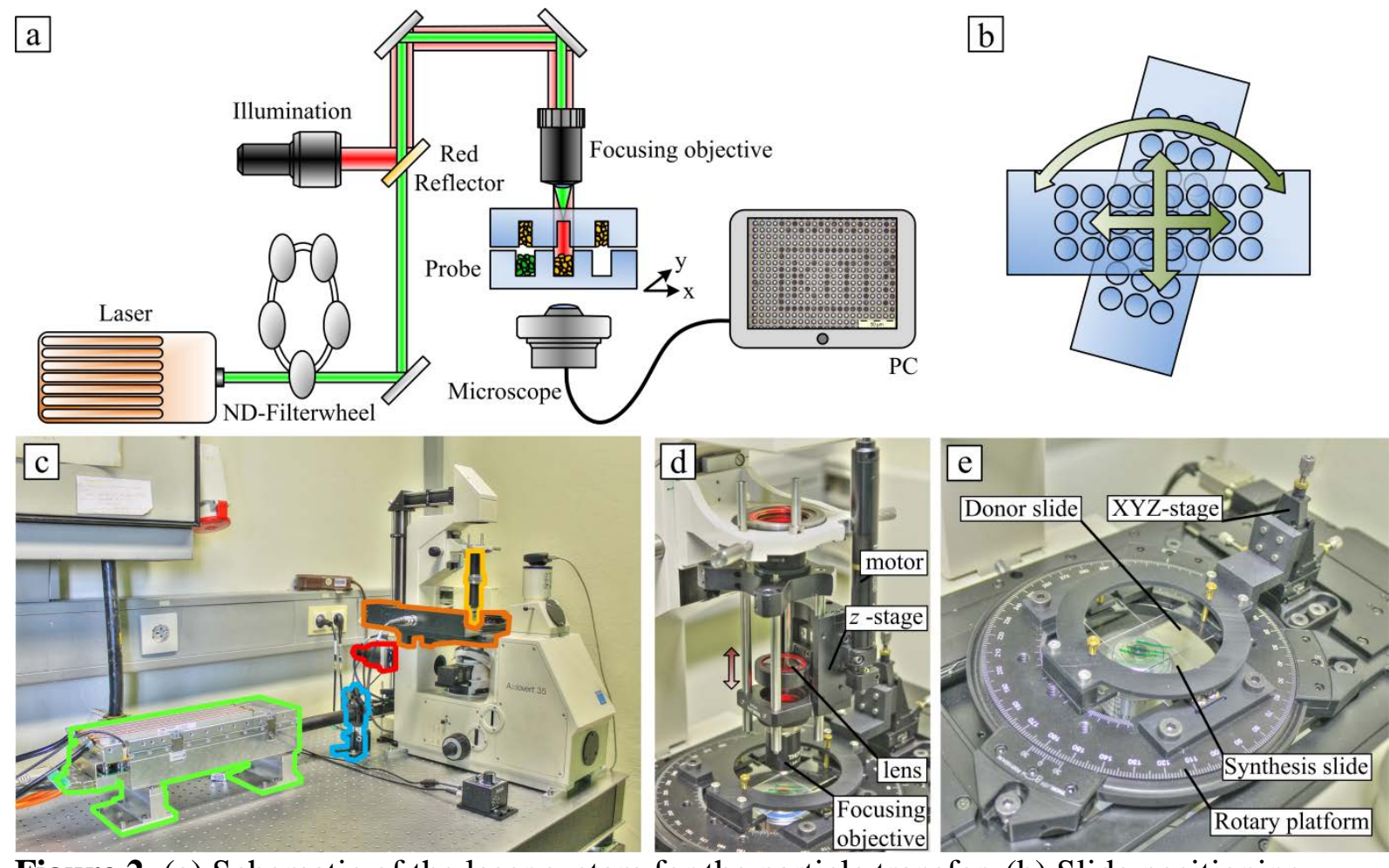

Figure 2. (a) Schematic of the laser system for the particle transfer. (b) Slide positioning system for aligning the donor slide relatively to the synthesis slide. Three degrees of freedom are used: one rotational and two translational degrees of freedom. (c) Photograph of the setup with the laser (green), neutral density filters (blue), light source (red), focusing device (yellow) and the microscopic stage (orange). (d) Photograph of the automated focusing device with the motor-driven movable lens. (e) Photograph of the slide positioning system. The alignment is realized by a rotary platform, which carries the synthesis slide, and a XYZ-stage which holds the donor slide. 

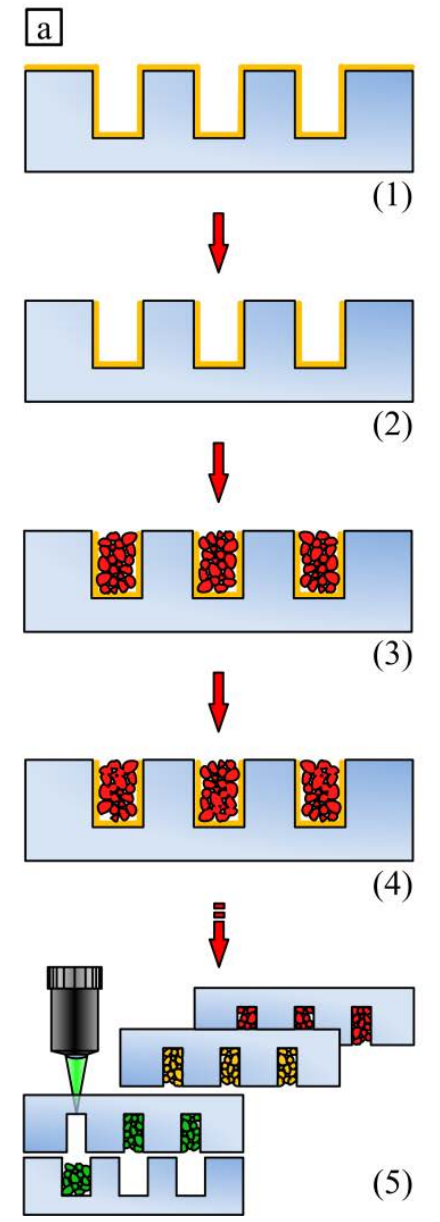
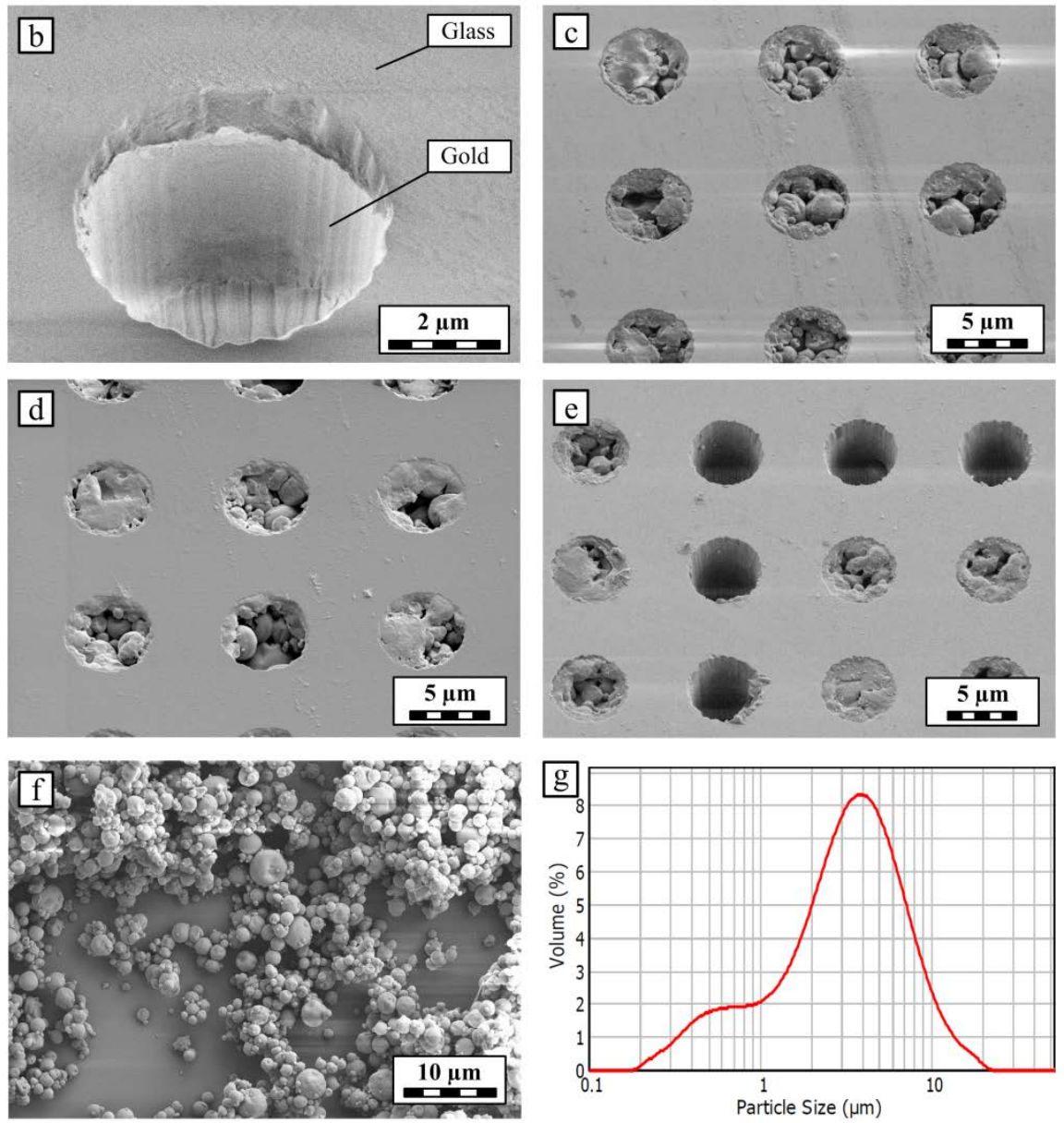

Figure 3. Preparation of the donor slide. (a) A structured glass slide is covered with a sputtered gold layer (a1). The gold is removed from the top surface using a lint-free tissue (a2). Filling the cavities with particles (a3). Sintering of the single particles to agglomerates in an oven at $90{ }^{\circ} \mathrm{C}$ for $15 \mathrm{~min}$ (a4). Multiple donor slides are used for the transfer (a5). (b) Scanning electron microscope image of a donor cavity with the gold layer before the particles are filled in. Filled donor cavities before (c) and after (d) the sintering process. (e) Emptied donor cavities after the transfer. (f) Scanning electron microscope image of the spherical microparticles used for the method described g) Size distribution of the microparticles. 

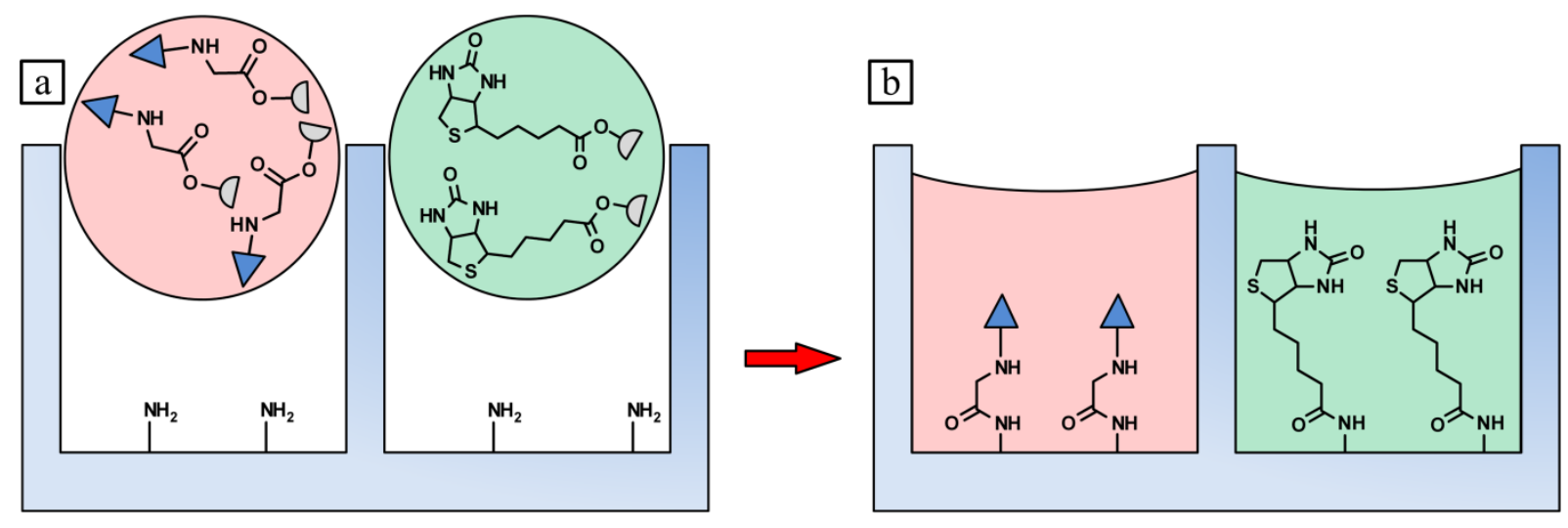

d

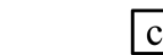

c
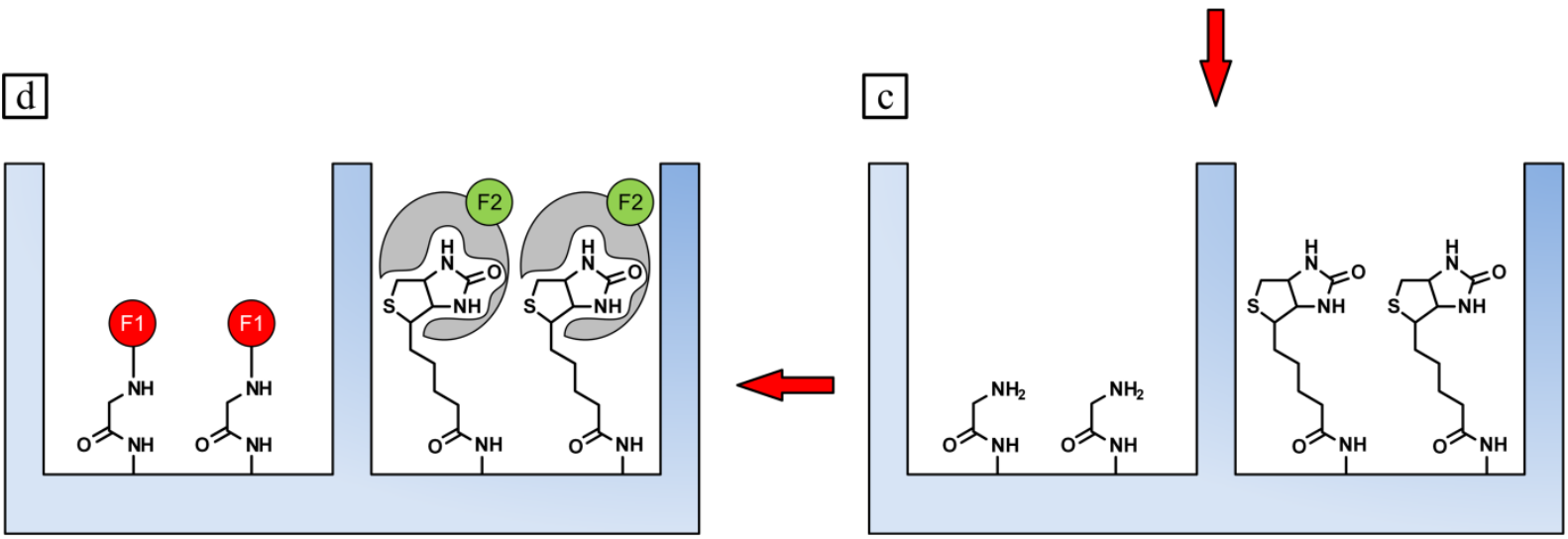

Figure 4. Particle based coupling of monomers. (a) Particle deposition with the described methods. (b) Melting of particles in an oven and coupling of monomers to the surface. (c) Washing and deprotection of monomers. (d) Fluorescent staining of coupled monomers over the free amino groups (left) and streptavidin (right). Blue triangle: Fmoc protection group; gray semicircle: OPfp activation group; F1, F2: fluorescent dyes 1 and 2. 

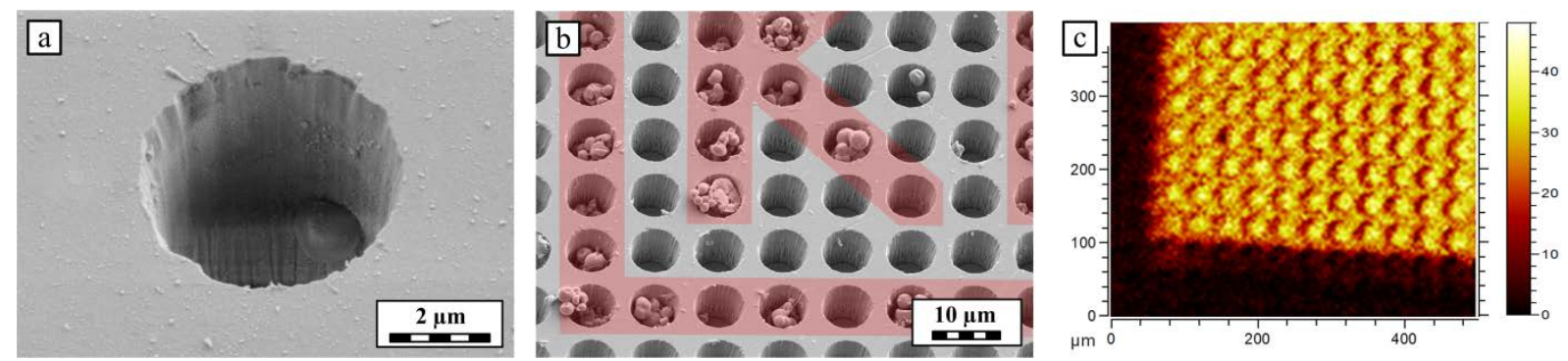

Figure 5. Transfer result. (a) Scanning electron microscope image of an emptied donor cavity. All particles as well as the gold layer are removed completely (diameter $7 \mu \mathrm{m}$ ). (b) Scanning electron microscope image of the synthesis slide with transferred particles forming the "KIT"letters (spot density 1,000,000 $\mathrm{cm}^{-2}$ ). (c) Secondary ion mass spectrometry (SIMS) measurement of a synthesis slide shows the homogeneous presence of gold surrounding the synthesis cavities. An area of 13 by 13 cavities was filled with particles using the transfer method \#2. 

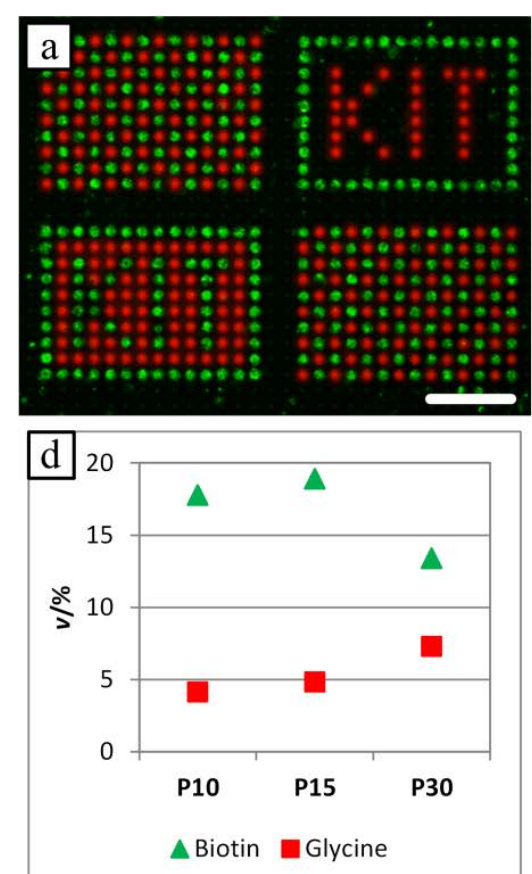
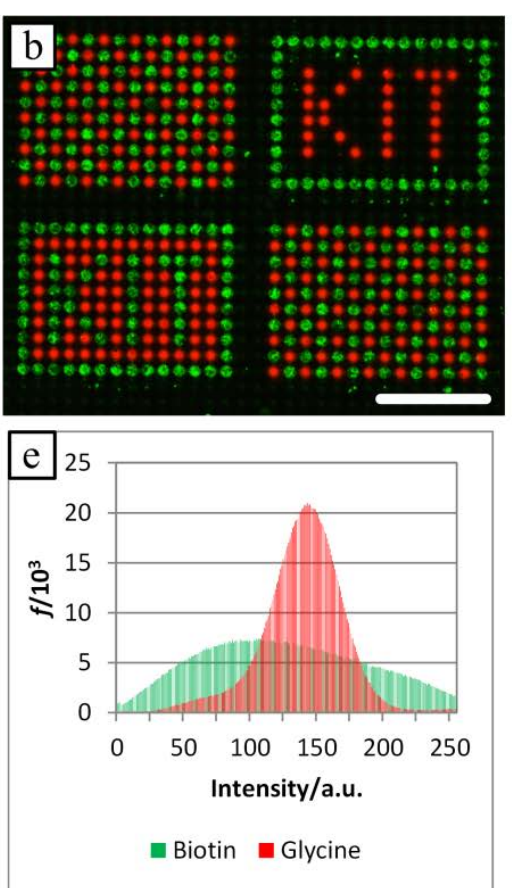
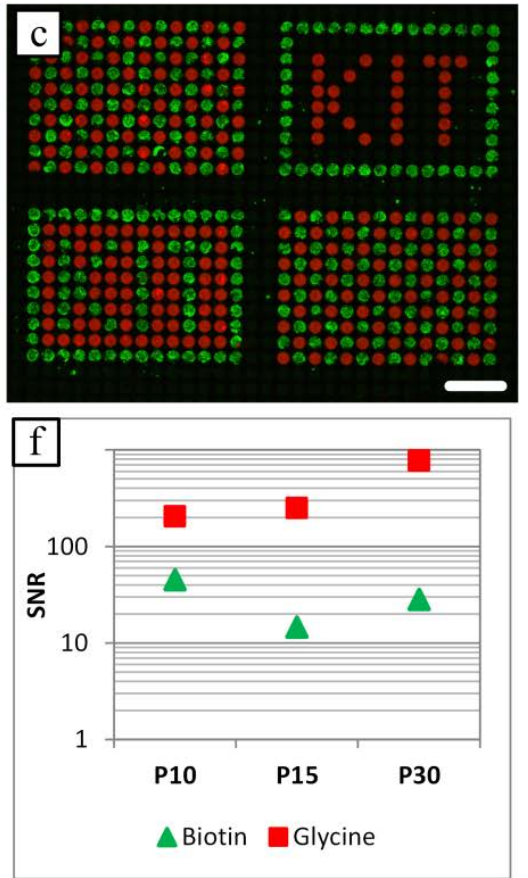

Figure 6. Fluorescent images and statistical data analysis. $(\mathrm{a}-\mathrm{c})$ Fluorescent images of the synthesis slide with spot densities of 1,000,000, 444,444 and 111,111 cm $\mathrm{cm}^{-2}$ (scale bars: $50 \mu \mathrm{m}$ (a) and $100 \mu \mathrm{m}(\mathrm{b}+\mathrm{c})$ ). Red signals correspond to stained glycine; green signals correspond to stained biotin. (d) Fluorescent signal variation $v$ for each channel and each spot density. (e) Histogram of all spots in each channel show the inhomogeneity of biotin compared to the homogeneity of glycine. (f) Signal to noise ratio (SNR) for each channel and each spot density. The low SNR of biotin can be explained with the high fluorescence signal variation resulting from the low solubility of biotin in the particle matrix material which leads to inhomogeneous signals. 

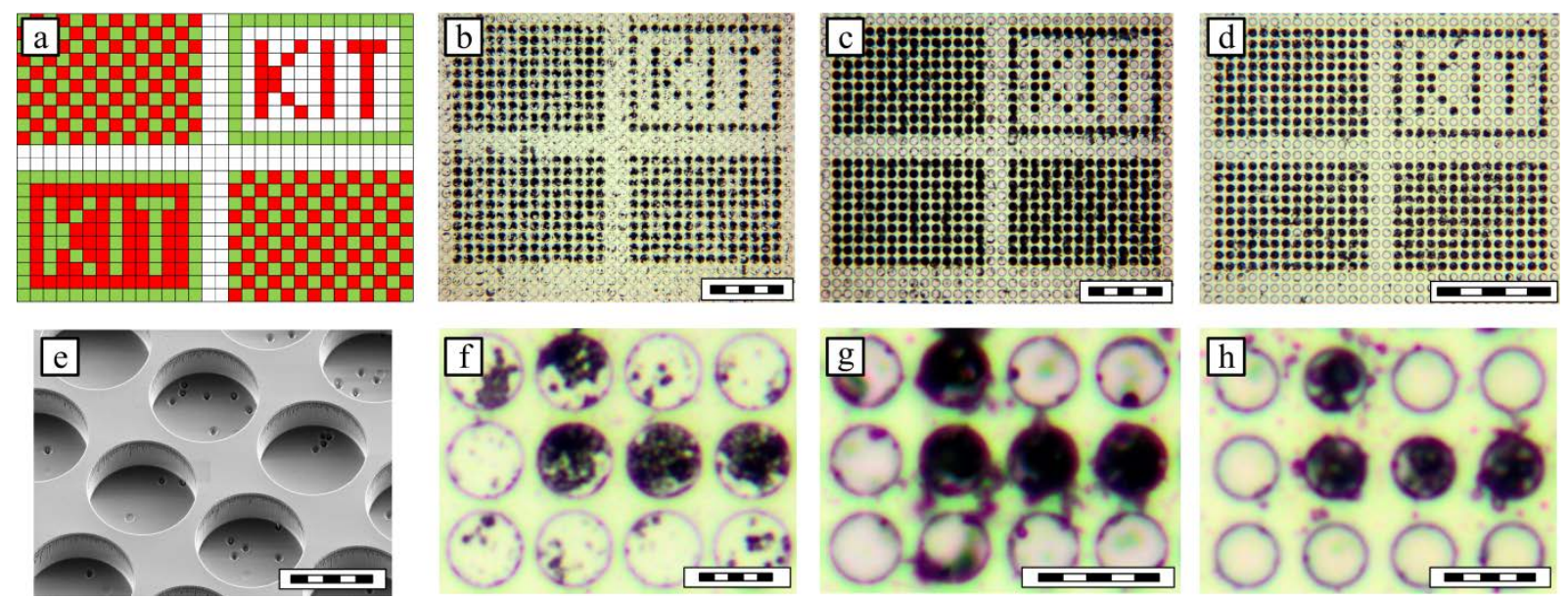

Figure 7. Reproducibility and scattering. (a) Predefined particle pattern. (b) - (d) Transferred particles with a spot density of 111,111 cm $\mathrm{cm}^{-2}$ (b) scale bar $\left.200 \mu \mathrm{m}\right), 444,444 \mathrm{~cm}^{-2}$ ((c) scale bar $100 \mu \mathrm{m})$ and 1,000,000 $\mathrm{cm}^{-2}((\mathrm{~d})$ scale bar $100 \mu \mathrm{m})$ used for the determination of the reproducibility. (e) Scanning electron microscope image of the substrate with the spot densities of $111,111 \mathrm{~cm}^{-2}$ (b) and $444,444 \mathrm{~cm}^{-2}$ (c) with visible defects resulting from the etching process in the range of the particle diameter (scale bar $20 \mu \mathrm{m})$. (f) - (h) Fragment of a corner with a spot density of 111,111 cm $\mathrm{cm}^{-2}$ (scale bar $30 \mu \mathrm{m}$ ), 444,444 $\mathrm{cm}^{-2}$ (scale bar $20 \mu \mathrm{m}$ ) and $1,000,000 \mathrm{~cm}^{-2}$ (scale bar $10 \mu \mathrm{m}$ ) used to analyze the scattering. 
The table of contents entry should be 50-60 words long, and the first phrase should be bold.

We present a method for the selective functionalization of microcavities with a density of up to 1 million microcavities per $\mathbf{c m}^{2}$ using two different transfer strategies. Polymer microparticles with embedded pre-activated monomers are selectively transferred into microcavities with laser radiation. Using Merrifield's solid phase synthesis, these monomers can be attached to the chemically modified surface.

Keywords: laser, microstructures, functional microparticles, functionalization, solid phase synthesis

Clemens von Bojnicic-Kninski, Valentina Bykovskaya, Frieder Maerkle, Roman Popov, Andrea Palermo, Daniela S. Mattes, Laura K. Weber, Barbara Ridder, Tobias C. Foertsch, Alexander Welle, Felix F. Loeffler, Frank Breitling, and Alexander Nesterov-Mueller*

Selective Functionalization of Microstructured Surfaces by Laser-Assisted Particle Transfer

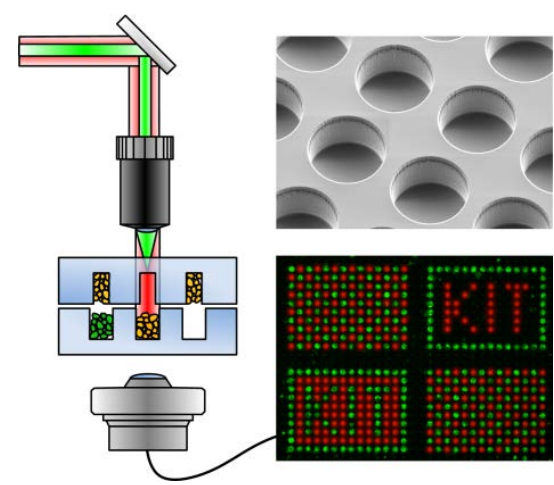

